\title{
The Clinical Significance of Vascular Factors in Glaucoma
}

\author{
Brent Siesky, Alon Harris, Nisha Kheradiya, Carlos Rospigliosi, Lynne McCranor, Rita Ehrlich
}

Department of Ophthalmology, Indiana University School of Medicine, Indianapolis, Indiana, USA

\section{INTRODUCTION}

Primary open angle glaucoma (OAG) is a multi-factorial optic neuropathy characterized by progressive retinal ganglion cell death and associated visual field loss. Glaucoma prevalence is $0.7 \%$ among 40-49 year olds and rises over subsequent decades to $7.7 \%$ amongst those over 80 years of age. ${ }^{1}$ Due to the aging of the United States population, the number of patients with OAG is expected to increase by $50 \%$ to 3.36 million in the year $2020 .^{2}$ OAG therefore represents an emerging disease with increasing cost and negative visual function impacts; yet our understanding of OAG risk factors and treatments are not fully apparent.

The only treatable risk factor for glaucoma is elevated intraocular pressure (IOP). Despite the medical lowering of IOP, however, some glaucoma patients continue to experience disease progression and subsequent irreversible blindness. For instance, in the early manifest glaucoma trial (EMGT) the disease progression rate in the treatment group was $45 \%$ as compared to $62 \%$ in the control arm. ${ }^{3}$ In the collaborative initial glaucoma treatment study (CIGTS) substantial visual field (VF) loss occurred in $10-13.5 \%$ of participants during 5 years of followup. ${ }^{4}$ Specifically, increased incidence of visual field deterioration occurred with older age (increased risk of VF loss by $40 \%$ every 10 years), race (nonwhites had a 50\% increased risk relative to whites) and diabetes (59\% increased risk relative to non-diabetic patients). ${ }^{4}$ In a similar manner, $20 \%$ of normal tension glaucoma (NTG) patients show continued visual field loss even after 5 years of IOP reduction treatment. ${ }^{5}$ Although IOP is still considered the only OAG risk factor, it is clear that glaucoma is a complex disease that is not prevented or cured by using only IOP reducing therapies in all patients. Among the other OAG risk factors, compromised ocular blood flow and faulty vascular autoregulation continue to emerge as possible contributors to OAG disease pathophysiology.

Vascular related OAG risk factors have been found in prospective, retrospective and large epidemiological studies throughout the world. Growing evidence suggests that glaucoma patients have reduced blood flow to the retina, choroid and optic nerve. It is unclear whether there is a primary contributing vascular component that promotes damage to the optic nerve and retinal ganglion cells (as well as aqueous humor dysregulation), if the OAG vascular component is secondary to increased IOP, or if it represents a combination of the two including impaired autoregulation within the OAG population. ${ }^{6}$ Several of the most common vascular risk factors which may be related to OAG disease include: aging, systemic blood pressure, nocturnal hypotension, ocular perfusion pressure, migraine, disk hemorrhage, diabetes and directly assessed reductions of ocular blood flow. Each of these topics will be reviewed in detail within this manuscript.

\section{AGING}

In population-based studies age is reported as an important risk factor for glaucoma. ${ }^{7}$ Age leads to many changes that can contribute to glaucoma which include vascular and mechanical changes as well as small increases in IOP. ${ }^{8}$ Mean IOP and cupto-disc ratio has been associated with age, especially in OAG patients over 75 years of age. ${ }^{9}$ Similarly, reduction of vascular function with age is well known and reduced choroidal and retrobulbar blood flow have been reported to occur with increasing age. ${ }^{10}$ The optic nerve head $(\mathrm{ONH})$ is primarily perfused by the short posterior ciliary arteries with some contribution from the retinal arterioles to the superficial nerve fiber layer. Studies in normal healthy individuals have shown reductions in blood flow to occur to the optic nerve with increasing age. ${ }^{11,12}$ Specifically, decreased blood flow was observed with age in the area of the neuroretinal rim and lamina cribrosa. ${ }^{13}$ However, aging also contributes to mechanical weakness in the area of the lamina cribrosa that can be further compromised by increased IOP. Over time there is an increase in elastin fiber coupled with increases in the connective tissue thickness of the optic nerve ${ }^{14}$ and increased thickness of the lamina cribrosa. ${ }^{15}$ Furthermore, there is a decrease in number of axons and thinning of the nerve fiber layer. ${ }^{16}$ The overall effects of aging are complex and hard to individually separate. The loss of normal vascular function with age seems to contribute to the overall age-related breakdown of eye structures and function. These age-related changes may disrupt the normal 
vascular regulation of ocular blood flow. For instance, vascular autoregulation dysfunction in response to postural induced changes in ocular perfusion pressure has been shown in glaucoma patients compared to healthy controls. ${ }^{17}$ Autoregulation of blood flow being impaired alone and/or in combination with elevated IOP may be compounding the damage to the optic nerve and contributing to visual field loss in OAG. ${ }^{18}$

\section{Systemic Blood Pressure and Perfusion Pressure}

Systemic hypertension ${ }^{19}$ and hypotension ${ }^{20}$ have both been reported as potential risk factors in glaucoma. It has been suggested that chronic hypertension may cause microvascular damage as a result of increased peripheral resistance in the small vessels and atherosclerosis changes, while hypotension may chronically reduce local ocular perfusion. It is important to understand that IOP and blood pressure determine ocular perfusion pressure (OPP) as OPP equals 2/3 (mean arterial pressure) minus IOP. ${ }^{21}$ This is also often further broken down into systolic and diastolic perfusion pressures. Patients with systolic blood pressure higher than $130 \mathrm{mmHg}$ or diastolic perfusion pressure (diastolic blood pressure minus IOP) lower than $30 \mathrm{mmHg}$ were reported to have higher risk for OAG. ${ }^{22}$ In the Egna-Neumarkt study ${ }^{23}$ a positive correlation was found between systemic blood pressure and diagnosis of OAG as well as to IOP. Reduced diastolic perfusion pressure below 55 $\mathrm{mmHg}$ is emerging as an important risk factor for OAG. Specifically, Hulsman et $\mathrm{al}^{24}$ recently reported an association between high tension glaucoma and high pulse pressure accompanied by arterial stiffness as well as with low diastolic perfusion pressure in patients receiving treatment for systemic hypertension. The increased vascular stiffness may contribute to the impaired autoregulation in glaucoma patients. Patients with increased diastolic blood pressure of $85 \mathrm{mmHg}$ or higher had a higher risk of NTG and surprisingly patients with low diastolic perfusion pressure had a significantly lower risk of NTG. Importantly, perfusion pressure fluctuations have been recently shown to be related to visual field progression. ${ }^{25}$

The information available on blood pressure and OAG continues to be complex, and often appears different among differing races. A population-based study on Hispanic subjects did not find a clear correlation between systemic hypertension and prevalence of OAG but did find that the lower the diastolic perfusion pressure the more likely the subject was to have OAG. ${ }^{26}$ Importantly, those with a perfusion pressure lower than $50 \mathrm{mmHg}$ had a 4 times greater risk of OAG than those with a perfusion pressure of $80 \mathrm{mmHg}{ }^{26}$ The Barbados Incidence Study of Eye Diseases (BISED) ${ }^{27}$ in a large, predominantly African-origin population, found that persons with hypertension had a statistically significant decreased risk of OAG. It can be hypothesized that higher blood pressure in the early stages can protect the optic nerve by maintaining the ocular perfusion pressure. OAG incidence was highest with low ocular perfusion pressure; lower systolic perfusion pressure more than doubled the relative risk (RR) and lower diastolic perfusion pressure (DPP) ( $<55 \mathrm{mmHg}$ ) more than tripled the RR of OAG. McLeod et $\mathrm{al}^{28}$ found a positive correlation between change in IOP from one year to the other and the change in systemic blood pressure during the same period. The Rotterdam study ${ }^{29}$ reported a similar association between systolic and diastolic blood pressure and IOP. Furthermore, hypertension was associated with high tension glaucoma but not with NTG. NTG patients were more likely to have decreased blood pressure, perhaps further emphasizing the role of defective perfusion pressures in this form of glaucoma. $^{30,31}$

Within the multitude of differing results on blood pressure and OAG, understanding how OAG patients may differ from healthy subjects in response to blood pressure changes may hold the key. As abnormal autoregulation has been reported in glaucoma patients, we need to answer whether a blood pressurerelated decrease in ocular perfusion pressure has a direct effect on ocular blood flow. Gherghel et al have reported a lower mean blood pressure and lower end diastolic velocity in the central retinal artery (CRA) in patients with progressing OAG compared to controls. ${ }^{32}$ Mean perfusion pressure correlated positively with end diastolic velocity in the ophthalmic artery (OA) and CRA and negatively with Pourcelot's resistive index in the OA and CRA. ${ }^{32}$ This suggests that OAG patients' ocular blood flow is compromised during changing blood pressure, IOP and perfusion pressure while healthy individuals can maintain constant ocular circulation.

\section{Nocturnal Hypotension}

It is well-established that circadian rhythms play an important role in maintaining homeostasis in the human body. In that capacity, blood pressure may be involved in OAG progression, not due to the overall level of blood pressure, but to an abnormal reduction in blood pressure during non-waking hours. It has been known for over four decades that $\mathrm{IOP}^{33}$ varies throughout the day, but not until more recently has it been shown that other factors affecting the eye such as systemic blood pressure, ${ }^{34}$ ocular perfusion pressure ${ }^{35}$ and ocular blood flow ${ }^{36}$ also follow circadian patterns. Studies indicate that blood pressure normally decreases at night, during sleep, to levels 10 to 20 percent below the diurnal average. ${ }^{37,38}$ It is also thought that perfusion instability, rather than a steady reduction of ocular blood flow, might contribute to glaucomatous optic neuropathy. ${ }^{39}$ Given the known circadian nature of systemic arterial pressure, numerous studies in recent years have attempted to associate glaucoma risk with nocturnal variations in blood pressure.

Hayreh et $\mathrm{al}^{40}$ performed 24-hour blood pressure monitoring on 166 white patients with either anterior ischemic optic 
neuropathy, NTG or OAG. Results showed that NTG patients had significantly lower diastolic blood pressure at night and a significantly greater mean decrease in diastolic blood pressure at night compared to patients with anterior ischemic optic neuropathy. Of particular interest was their finding of an apparent interaction between systemic hypertension, deterioration of visual field and nocturnal blood pressure. Their results showed that among patients taking systemic hypotensive medication, the nocturnal blood pressure dips were significantly larger for those with progressing visual field loss than compared to those who had clinically stable visual fields. This progressing visual field group also showed a significantly lower mean minimum nighttime systolic blood pressure as compared to the stable group. A more recently published paper $^{41}$ also suggests an association between nocturnal blood pressure levels and progressing visual field damage. Twentyfour hour ambulatory blood pressure readings were taken for three groups: NTG, OAG and age-matched normal controls. The glaucoma patients with progressing visual field loss had significantly lower mean nighttime blood pressure and significantly greater mean decrease in nighttime blood pressure than the patients with stable visual fields.

These data indicate a possible association between systemic blood pressure and glaucoma; however the effect this factor may have on ocular circulation is not fully known. As IOP, blood pressure and perfusion pressure are now known to fluctuate during the day and night, previously unseen mild chronic hypo-perfusion of the $\mathrm{ONH}$ and retina during these fluctuations may contribute to OAG pathophysiology.

\section{Other Vascular Risk Factors: Migraine, Disk Hemorrhage, Diabetes}

Migraine as a disease may reflect a more generalized vasospastic tendency that can further affect ocular blood flow. For instance, migraine has been reported to influence the survival time of patients with NTG. The reported risk ratio of patients with migraine to experience disease progression was 2.58 times greater than patients without migraine. ${ }^{42}$ Migraine is significantly more common in patients with NTG compared with control subjects and patients with high-pressure glaucoma. ${ }^{43}$ In the NTG study, untreated female patients with migraine visual function deteriorated faster than those without migraine. ${ }^{47}$ Migraine may therefore represent an larger vascular problem that appears to contribute to OAG disease.

Disk hemorrhages have also been positively related to NTG. Patients with presence of disk hemorrhage at baseline had 2.7 times greater risk to progress than patients without hemorrhage at baseline. ${ }^{42}$ Rasker et $\mathrm{al}^{44}$ reported that $80-89 \%$ of glaucoma patients with optic disc hemorrhages experienced glaucomatous progression as opposed to $32 \%$ of patients who did not have disc hemorrhage (mean follow-up of 9 years). Further, OAG patients with diabetes and those using aspirin were reported to have a higher risk of having optic disc hemorrhage. ${ }^{45}$ The early manifest glaucoma trial also reported that patients with frequent disc hemorrhages had increased progression of glaucoma. ${ }^{46}$ It was also reported that NTG patients without baseline disc hemorrhage responded better to treatment then patients with baseline disc hemorrhage. $^{47}$

Diabetes, a disease with many vascular complications, has been reported to be related to glaucoma. ${ }^{48}$ However the evidence for diabetes as a contributing factor in OAG pathology is currently lacking. Population-based studies, such as the Baltimore eye survey, ${ }^{49}$ the Barbados eye study ${ }^{7}$ and the Rotterdam study ${ }^{50}$ have failed to support an association between diabetes and glaucoma. Due to the multitude of vascular changes in diabetes, more prospective evidence is required before definitive conclusions can be made.

\section{Directly Assessed Reductions of Ocular Blood Flow}

Reduced ocular perfusion may be secondary to IOP elevation or represent a primary insult to the optic nerve in glaucoma. Chronic ocular ischemia may be due to faulty vascular autoregulation and the inability of the vasculature to overcome elevated IOP to maintain adequate perfusion. Regardless, chronic optic nerve ischemia has been shown to induce retinal ganglion cell loss independent of IOP. ${ }^{51}$ The cascade of events involving reduction of ocular blood flow leading to retinal ganglion cell apoptosis are shown in Figure 1. ${ }^{52}$

There is a multitude of various small prospective studies which have demonstrated blood flow deficiencies of OAG patients in the retinal, ${ }^{53}$ choroidal $^{54}$ and retrobulbar ${ }^{55-58}$ circulations. Reductions in ocular blood flow have also been shown to correspond with areas of glaucomatous visual field loss. ${ }^{59}$ The studies and evidence mentioned above utilize many different ocular blood flow imaging technologies in an attempt to reveal blood flow deficits in glaucoma. Each imaging technology has various limitations and is tissue-specific within

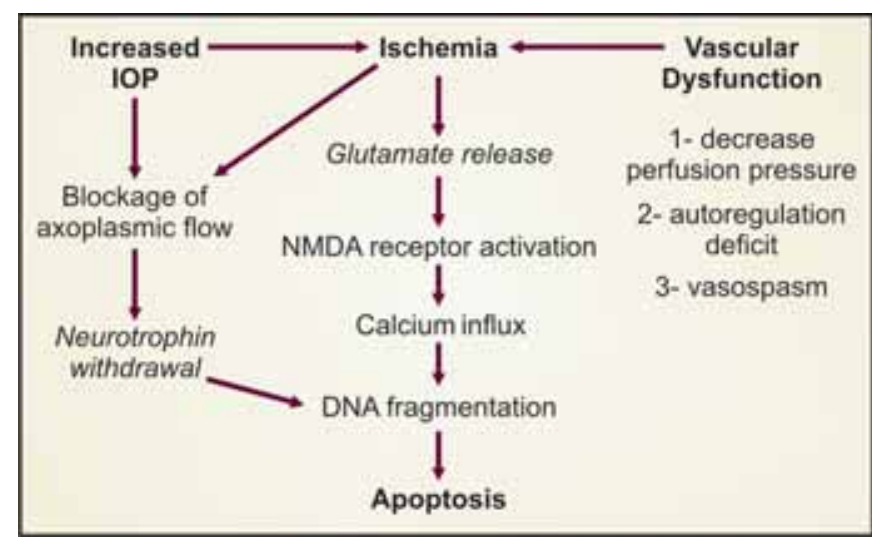

Fig. 1 
the eye's vasculature and is discussed in great detail elsewhere. ${ }^{60}$ The overwhelming amount of evidence from the various imaging techniques in the retinal, choroidal and retrobulbar vascular beds, however, points to ischemia's role in OAG pathophysiology. Moreover, it is important to consider that the direct and indirect evidence for ocular ischemia in OAG represents a surrogate for retinal metabolism and oxygenation.

The direct measurement of retinal tissue oxygenation would reveal the true nature of ischemia's impact on retinal ganglion cell health and function. Currently, ocular blood flow investigations suggest that retinal tissue may be experiencing a lack of oxygenation and this hypoxia is contributing to OAG disease. New and emerging metabolic assessment tools may help reveal how reductions in ocular blood flow and ocular tissue hypoxia are related. ${ }^{61}$ Specifically, one study by Michelson et al measured the oxygen saturation in retinal arterioles and venules in patients with glaucomatous optic neuropathy by imaging spectrometry. ${ }^{62}$ In all examined eyes, the arteriolar oxygen saturation and the retinal arterio-venous differences in oxygen correlated significantly with the patient's rim area. Eyes with NTG but not OAG showed significantly decreased arteriolar oxygen saturation. These metabolic assessment tools, while still undergoing improvements, ${ }^{63}$ may provide the next step in specifically assessing retinal hypoxia, possibly as a result of ocular ischemia.

\section{Summary}

As of today, IOP remains the only treatable risk factor in glaucoma patients. We currently have a variety of topical and systemic medications, and various surgeries to effectively lower a patient's IOP. Although a large percentage of patients respond to IOP lowering treatment showing slower deterioration of their visual function, we still face two challenging groups of patients. The first group, are patients that do not respond well to IOP reduction despite medical and surgical treatment and the other group are patients that continue to deteriorate despite wellcontrolled IOP. Understanding these risk factors that can be responsible for the continued deterioration while addressing and manipulating the risk factors may improve the outcome of OAG patients. Vascular risk factors, including faulty autoregulation of the ocular vasculature, continue to emerge as important considerations in glaucoma. Early pilot data suggests that vascular abnormalities are related to visual field and optic nerve damage. ${ }^{64,65}$ These pilot studies suggest that compromised ocular blood flow may occur before glaucomatous changes are detectable from visual field testing. It is important to note, however, that only after a longitudinal, comprehensive and statistically justified study shows that glaucomatous disease progression is related to ocular blood flow can we be confident in changing treatment direction to vascular risk factors. It remains to be seen if changing possible vascular risk factors will indeed change patients' structural as well as functional outcomes. Future treatment approaches should target increasing ocular blood flow, elevating and maintaining ocular perfusion pressure, moderating diurnal fluctuations in blood pressure, IOP and perfusion pressure and providing direct neuroprotection.

\section{REFERENCES}

1. National Eye Institute. Statistics and Data. Prevalence of Blindness Data. Data Tables. http://www.nei.nih.gov/eyedata/ pbd_tables.asp. Accessed on September 18, 2007.

2. Eye Diseases Prevalence Research Group. Prevalence of openangle glaucoma among adults in the United States. Arch Ophthalmol 2004;122:532-8.

3. Heijl A, Leske MC, Bengtsson B, et al. Early Manifest Glaucoma Trial Group. Reduction of intraocular pressure and glaucoma progression: results from the Early Manifest Glaucoma Trial. Arch Ophthalmol 2002;120:1268-79.

4. Lichter PR, Musch DC, Gillespie BW, et al. Interin clinical outcome in the collaborative initial glaucoma treatment study comparing initial treatment randomized to medications or surgery. Ophthalmology 2001;108:1943-53.

5. Collaborative Normal-Tension Glaucoma Study Group. The effectiveness of intraocular pressure reduction in the treatment of normal-tension glaucoma. Am J Ophthalmol 1998;126:498505.

6. Flammer J, Orgül S, Costa VP, et al. the impact of ocular blood flow in glaucoma. Progress in retinal and eye research 2002;21:259-393.

7. Leske MC, Connell AMS, Wu SY, et al. the Barbados eye study group. Risk factors for open angle glaucoma. Arch ophthalmol 1995;113:918-24.

8. Colton T, Ederer F. The distribution of intraocular pressure in the general population. Surv Ophthalmol 1980;25:123-9.

9. Klein BE, Klein R, Lee KE, Hoyer CJ. Does the intraocular pressure effect on optic disc cupping differ by age? Trans Am Ophthalmol Soc 2006;104:143-8.

10. Harris A, Harris M, Biller J, et al. Aging affects the retrobulbar circulation differently in women and men. Arch ophthalmol 2000;118:1076-80.

11. Boehm AG, Koeller AU, Pillunat LE. The effect of age on optic nerve head blood flow. Invest Ophthalmol Vis Sci 2005;46: 1291-5.

12. Rizzo JF, Feke GT, Goger DG, et al. Optic nerve head blood speed as a function of age in normal human subject. Invest Ophthalmol Vis Sci 1991;32:3263-72.

13. Embleton SJ, Hosking SL, Roff Hilton EJ, Cunliffe IA. Effect of senescence on ocular blood flow in the retina, neuroretinal rim and lamina cribrosa, using scanning laser Doppler flowmetry. Eye. 2002;16:156-62.

14. Moya FJ, Brigatti L, Caprioli J. Effect of aging on optice nerve appearance: a longitudinal study. Br J Ophthalmol 1999; 83:56772.

15. Kotecha A, Izadi S, Jeffery G. Age-related changes in the thickness of the human lamina cribrosa. Br J Ophthalmol 2006;90: 1531-4.

16. Da Pozzo S, Iacono P, Marchesan R, et al. The effect of ageing on retinal nerve fibre layer thickness: an evaluation by scanning 
laser polarimetry with variable corneal compensation. Acta Ophthalmol Scand. 2006;84:375-9.

17. Feke GT, Pasquale LR. Retinal Blood Flow Response to Posture Change in Glaucoma Patients Compared with Healthy Subjects. Ophthalmology 2007;7; [Epub ahead of print]

18. Flammer J, Haefliger IO, Orgul S and Resink T. Vascular dysregulation: a principal risk factor for glaucomatous damage? J Glaucoma 1999;8:212-9.

19. Wong T, Mitchell P. The eye in hypertension. Lancet 2007;369: 425-35.

20. Hayreh SS. Role of nocturnal arterial hypotension in the development of ocular manifestations of systemic arterial hypertension. Curr Opin Ophthalmol 1999;10:474-82.

21. Harris A, Kagemann L, Cioffi G. Assessment of Human Ocular Hemodynamics. Surv Ophthalmol 1998;42:509-33.

22. Tielsh JM, Katz J, Sommer A, et al. Hypertension, Perfusion Pressure, and Primary Open-angle Glaucoma. Arch Ophthalmol 1995;113:216-21.

23. Bonomi L, Marchini G, Marraffa M, et al. Vascular risk factors for primary open angle glaucoma. The Egna-Neumarkt study. Ophthalmology 2000;107:1287-93.

24. Hulsman CAA, Vingerling JR, Hofman A, et al. Blood pressure, arterial stiffness and open angle glaucoma. The Rotterdam study. Arch ophthalmol 2007;125:805-12.

25. Choi J, Jeong J, Cho HS, Kook MS. Effect of Nocturnal Blood Pressure Reduction on Circadian Fluctuation of Mean Ocular Perfusion Pressure: A Risk Factor for Normal Tension Glaucoma. Invest Ophthalmol Vis Sci 2006;47:831-6.

26. Quigley HA, West SK, Rodriguez J, et al. The prevalence of glaucoma in a population based study of Hispanic Subjects. Proyecto VER. Arch Ophthalmology 2001;119:1819-26.

27. Leske MC, Wu SY, Nemesure B, Hennis A. for the Barbados Eye Studies Group Incident Open-Angle Glaucoma and Blood Pressure. Arch Ophthalmol 2002;120:954-9.

28. Mcleond SD, West SK, Quigley HA, Fozard JL. A longitudinal study of the relationship between intraocular and blood pressure. Invest ophthalmol Vis Sci 1990;31:2361-6.

29. Dielemans I, Vingerling JR, Algra D, et al. Primary open-angle glaucoma, intraocular pressure, and systemic blood pressure in the general elderly population. The Rotterdam Study. Ophthalmology 1995;102:54-60.

30. Leighton DA, Phillips CI. Systemic Blood Pressure in OpenAngle Glaucoma, Low Tension Glaucoma, and the Normal Eye. Br J Ophthalmol 1972;56:447-53.

31. Flammer J. The vascular concept of glaucoma. Surv Ophthalmol 1994;38 Suppl:S3-6.

32. Gherghel D, Orgul S, Gugleta K, et al. Relationship between ocular perfusion pressure and retrobulbar blood flow in patients with glaucoma with progressive damage. Am J Ophthalmol 2000;130:597-605.

33. Drance SM. Diurnal variation of intraocular pressure in treated glaucoma. Arch Ophthalmol 1963;70:302-11.

34. O’Brien E, Murphy J, Tyndall A, et al. Twenty-four-hour ambulatory blood pressure in men and women aged 17 to 80 years: the allied Irish Bank Study. J Hypertens 1991;9:355-60.

35. Liu JH, Zhang X, Kripke DF, Weinreb RN. Twenty-four-hour intraocular pressure pattern associated with early glaucomatous changes. Invest Ophthalmol Vis Sci 2003;44:1586-90.

36. Osusky R, Rohr P, Schotzau A, Flammer J. Nocturnal dip in the optic nerve head perfusion. Jpn J Ophthalmol 2000;44:12831.

37. O’Brien E, Murphy J, Tyndall, et al. Twenty-four Hour Ambulatory Blood Pressure in Men and Women Aged 17 to 80 Years: The Allied Irish Bank Study. J Hypertension 1991;9:35560.

38. Staessen J, Pagard R, Lijnen P, et al. Mean and Range of the Ambulatory Pressure in Normotensive Subjects from a MetaAnalysis of 23 Studies. Am J Cardiology 1991;67:723-7.

39. Grieshaber MC, Flammer J. Blood flow in glaucoma. Curr Opin Ophthalmol 2005;16:79-83.

40. Hayreh SS, Zimmerman MB, Podhajsky P, Alward WL. Nocturnal Arterial Hypotension and its Role in Optic Nerve Head and Ocular Ischemic Disorders. Am J Ophthalmol 1994;117: 603-24.

41. Graham SL, Drance SM, Wijsman K, et al. Ambulatory Blood Pressure Monitoring in Glaucoma. Ophthalmology 1995;102:619.

42. Drance S, Anderson DR, Schulzer M. Risk factors for progression of visual field abnormalities in normal tension glaucoma. Am J Ophthalmol 2001;131:699-708.

43. Cursiefen C, Wisse M, Cursiefen S, et al. Migraine and tension headache in high-pressure and normal pressure glaucoma. Ophthalmol 1998;105:216-23.

44. Rasker MT, van den Enden A, Bakker D, Hoyng PF. Deterioration of visual fields in patients with glaucoma with and without optic disc hemorrhages. Arch Ophthalmol 1997;115:1257-62.

45. Soares AS, Artes PH, Andreou P, et al. Factors associated with optic disc hemorrhages in glaucoma 2004;111:1653-7.

46. Leske MC, Heijl A, Hyman L, et al. Factors for progression and glaucoma treatment: the Early Manifest Glaucoma Trial. Curr Opin Ophthalmol 2004;15:102-6.

47. Anderson DR, Drance SM, Schulzer M. Collaborative NormalTension Glaucoma Study Group. Factors that predict the benefit of lowering intraocular pressure in normal tension glaucoma. Am J Ophthalmol 2003;136:820-9.

48. Nielsen NV. The prevalence of glaucoma and ocular hypertension in type 1 and type 2 diabetes mellitus. Arch Ophthalmol 1983;61:662-72.

49. Tielsch JM, Katz J, Quigley HA, et al. Diabetes, intraocular pressure and primary open angle glaucoma in the Baltimore eye survey. Ophthalmology 1995;102:48-53.

50. de Voogd S, Ikram MK, Wolfs RC, et al. Is diabetes mellitus a risk factor for open-angle glaucoma? The Rotterdam Study. Ophthalmology 2006;113:1827-31.

51. Cioffi GA, Wang L, Fortune B, et al. Chronic ischemia induces regional axonal damage in experimental primate optic neuropathy. Arch Ophthalmol 2004;122:1517-25.

52. Harris A, Rechtman E, Siesky B, et al. The Role of OpticNerve Blood Flow in the Pathogenesis of Glaucoma. Ophthalmology Clinics of North America. 2005;18:345-53.

53. Chung HS, Harris A, Kagemann L, Martin B. Peripapillary retinal blood flow in normal tension glaucoma. $\mathrm{Br} \mathrm{J}$ Ophthalmol 1999;83:466-9. 
54. Yin ZQ, Vaegan, Millar TJ, et al. Widespread choroidal insufficiency in primary open-angle glaucoma. J Glaucoma 1997;6:23-32.

55. Butt Z, McKillop G, O’Brien C, et al. Measurement of ocular blood flow velocity using colour Doppler imaging in low tension glaucoma. Eye 1995;9:29-33.

56. Galassi F, Sodi A, Ucci F, et al. Ocular haemodynamics in glaucoma associated with high myopia. Int'l Ophthalmol 1998;22:299-305.

57. Harris A, Sergott RC, Spaeth GL, et al. Color Doppler analysis of ocular vessel blood velocity in normal-tension glaucoma. Am J Ophthalmol 1994;118:642-9.

58. Rojanapongpun P, Drance SM, Morrison BJ. Ophthalmic artery flow velocity in glaucomatous and normal subjects. $\mathrm{Br} \mathrm{J}$ Ophthalmol 1993;77:25-9.

59. Breil P, Krummenauer F, Schmitz S, Pfeiffer N. [The relationship between retrobulbar blood flow velocity and glaucoma damage.] [German] Ophthalmologe 2002;99:613-6.

60. Harris A, Jonescu-Cuypers CP, Kagemann L, et al. Atlas of ocular blood flow - vascular anatomy, pathophysiology, and metabolism. Philadelphia, Butterworth-Heinemann, 2003;19-70.

61. Harris A, Dinn RB, Kagemann L, Rechtman E. Review of methods for human retinal oximetry. Ophthalmic Surg Lasers Imaging 2003;34:152-64.

62. Michelson $\mathrm{G}$, Scibor M. Intravascular oxygen saturation in retinal vessels in normal subjects and open-angle glaucoma subjects. Acta Ophthalmol Scand 2006;84:289-95.
63. Hardarson SH, Harris A, Karlsson RA, Halldorsson GH, Kagemann L, Rechtman E, et al. Automatic retinal oximetry. Invest Ophthalmol Vis Sci 2006;47:5011-6.

64. Topouzis F, Coleman AL, Harris A, et al. Association of blood pressure status with the optic disc structure in non glaucoma subjects. The Thessaloniki Eye Study. Am J Ophthalmol 2006; 142:60-7.

65. Galassi F, Sodi A, Ucci F, et al. Ocular Hemodynamics and Glaucoma Prognosis: A Color Doppler Imaging Study. Arch Ophthalmol 2003;121:1711-5.

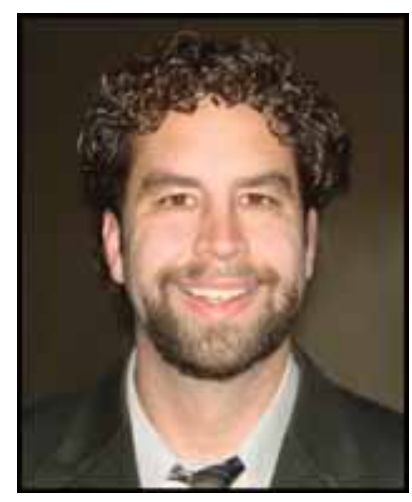

Dr Brent Siesky (bsiesky@iupui.edu)

“All men should strive to learn before they die, what they are running from, and to, and why"

— James Thurber 